\title{
A Nuclear RNA Degradation Pathway Helps Silence Polycomb/H3K27me3-Marked Loci in Caenorhabditis elegans
}

\author{
Anna Mattout, ${ }^{1,3}$ Dimos Gaidatzis, ${ }^{1}$ Véronique Kalck, ${ }^{1}$ and Susan M. Gasser ${ }^{1,2}$ \\ ${ }^{1}$ Friedrich Miescher Institute for Biomedical Research, CH-4058 Basel, Switzerland \\ ${ }^{2}$ University of Basel, Faculty of Science, CH-4056 Basel, Switzerland \\ Correspondence: susan.gasser@fmi.ch
}

\begin{abstract}
In fission yeast and plants, RNA-processing pathways contribute to heterochromatin silencing, complementing well-characterized pathways of transcriptional repression. However, it was unclear whether this additional level of regulation occurs in metazoans. In a genetic screen, we uncovered a pathway of silencing in Caenorhabditis elegans somatic cells, whereby the highly conserved, RNA-binding complex LSM2-8 contributes to the repression of heterochromatic reporters and endogenous genes bearing the Polycomb mark H3K27me3. Importantly, the LSM2-8 complex works cooperatively with a $5^{\prime}-3^{\prime}$ exoribonuclease, XRN-2, and disruption of the pathway leads to selective mRNA stabilization. LSM2-8 complex-mediated RNA degradation does not target nor depend on $\mathrm{H} 3 \mathrm{~K} 9 \mathrm{me} 2 / \mathrm{me} 3$, unlike previously described pathways of heterochromatic RNA degradation. Up-regulation of $l s m-8$-sensitive loci coincides with a localized drop in H3K27me3 levels in the $l s m-8$ mutant. Put into the context of epigenetic control of gene expression, it appears that targeted RNA degradation helps repress a subset of H3K27me3-marked genes, revealing an unappreciated layer of regulation for facultative heterochromatin in animals.
\end{abstract}

Genomic DNA is organized into highly condensed, dark-staining heterochromatin, which correlates with reduced gene expression (Trojer and Reinberg 2007; Wenzel et al. 2011; Saksouk et al. 2015), and less condensed open chromatin, which is transcribed. Whereas the primary mechanism for both constitutive (enriched for H3K9me2/3) and facultative (enriched for H3K27me3) heterochromatic gene repression is thought to involve the inhibition of RNA polymerases, robust pathways that silence at the co- and posttranscriptional levels have also been documented in fission yeast and plants (Buhler 2009; Wang et al. 2016).

A genome-wide screen in our laboratory that monitored the derepression of a heterochromatic, multicopy reporter in Caenorhabditis elegans embryos identified 29 factors essential for silencing. Most of these were chromatin modifiers and transcription-related proteins (Towbin et al. 2012) (Figs. 1A and 2A), but we also recovered three subunits of the evolutionarily conserved like-SM protein (LSM) complex (Fig. 1A). A role for these proteins in the repression of heterochromatin was unexpected given the reported roles for the two LSM complexes, LSM1-7 and LSM2-8, in general RNA metabolism but not in transcriptional control per se (Kufel et al. 2004; Beggs 2005; Tharun 2009; Perea-Resa et al. 2012; Golisz et al. 2013; Cornes et al. 2015). Whereas pathways of RNA processing and degradation were reported to contribute to silencing in fission yeast and plants (Buhler et al. 2007; Vasiljeva et al. 2008; Keller et al. 2012; Zofall et al. 2012; Yamanaka et al. 2013; Egan et al. 2014; Chalamcharla et al. 2015; Tucker et al. 2016), heterochromatic silencing through transcript degradation had not been documented in animals.

The RNA interference (RNAi) screen that identified gut-2/lsm-2, $l s m-5$, and $l s m-6$ monitored the robust derepression or desilencing of a $C$. elegans heterochromatic reporter, which consisted of an integrated array of several hundred copies of a green fluorescent protein (GFP)-encoding reporter gene driven from a ubiquitously active promoter (Towbin et al. 2012). In a copy-number-dependent manner, these heterochromatic reporters carry the histone modifications $\mathrm{H} 3 \mathrm{~K} 9 \mathrm{me} 2 / 3$ and $\mathrm{H} 3 \mathrm{~K} 27 \mathrm{me} 3$, but lack H3K 4 methylation, and are sequestered at the nuclear envelope, thereby mimicking many aspects of endogenous heterochromatin (Meister et al. 2010; Towbin et al. 2010). As expected, the loss of either of the two H3K9 methyltransferases (met-2 and set-25) or the EZH2 homolog mes2, which deposits $\mathrm{H} 3 \mathrm{~K} 27 \mathrm{me} 3$, led to reporter derepression (Towbin et al. 2012). It was surprising, however, to detect three subunits of an RNA processing complex with the same phenotype. Therefore, we examined in more detail how LSM proteins regulate gene expression within heterochromatic domains in C. elegans (Mattout et al. 2020).

\section{LSM PROTEINS REDUCE MRNA FROM HETEROCHROMATIC REPORTERS SELECTIVELY}

The initial screen for reporter derepression monitored GFP expression in embryos (Fig. 1B). The elimination of LSM proteins by $l s m-2, l s m-5$, or $l s m-6$ RNAi, on the other

\footnotetext{
${ }^{3}$ Present address: Université Paul Sabatier-CNRS UMR 5088, 31062 Toulouse, France

(C) 2019 Mattout et al. This article is distributed under the terms of the Creative Commons Attribution-NonCommercial License, which permits reuse and redistribution, except for commercial purposes, provided that the original author and source are credited.
} 
A

Integrated heterochromatic reporter

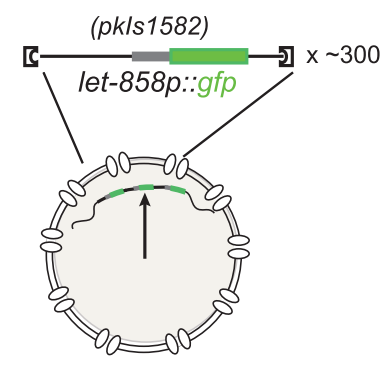

RNA processing hits:

$\begin{array}{ll}\text { gut-2 } & \text { LSM-2 } \\ \text { Ism-5 } & \text { LSM-5 } \\ \text { Ism-6 } & \text { LSM-6 }\end{array}$

C

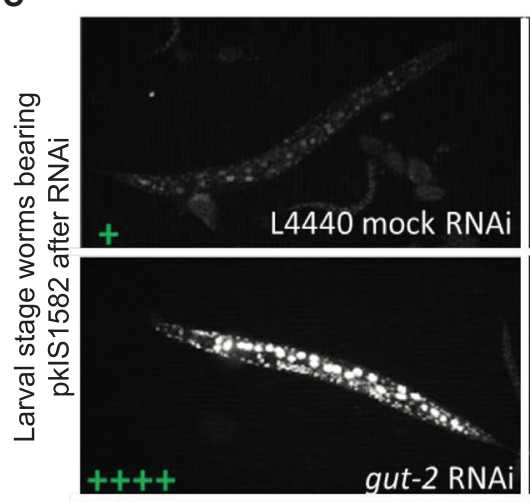

B

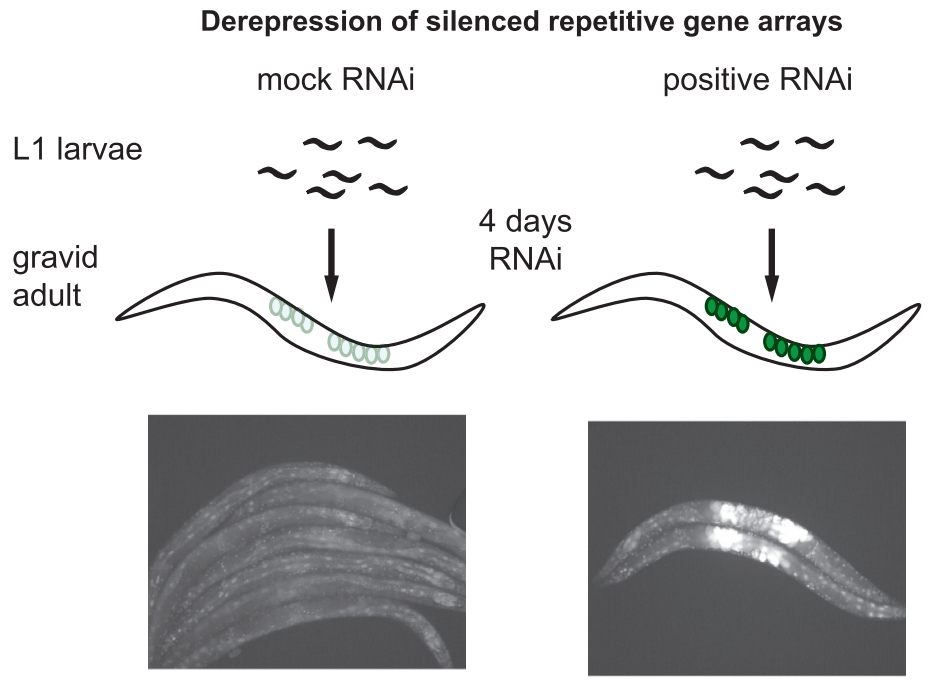

GW306 (let-858::GFP::let858 3'UTR; rol-6(su1006))
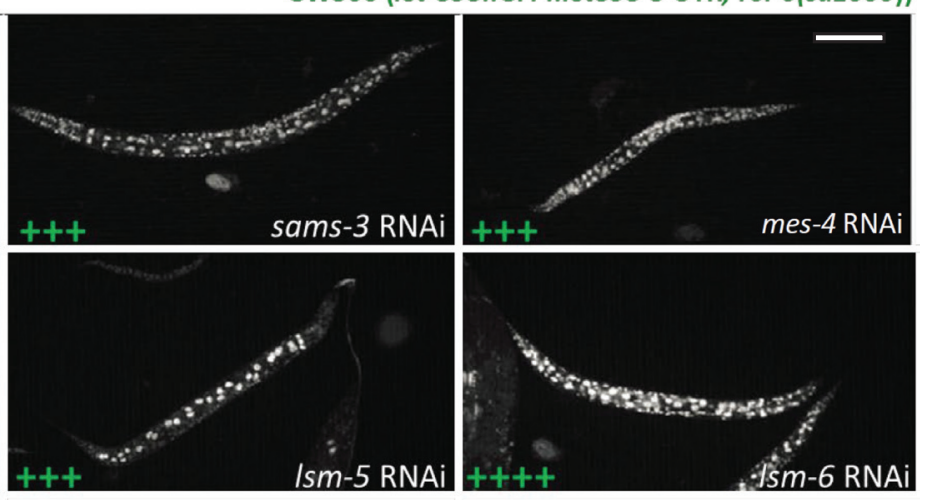

D

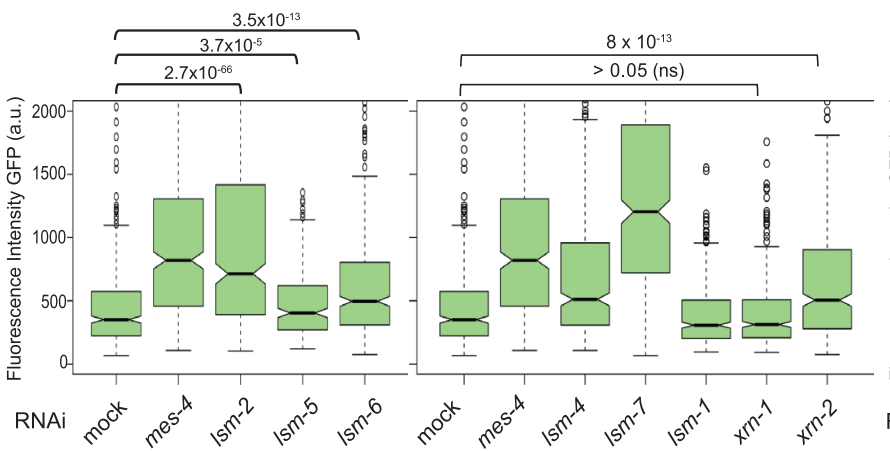

E

Heterochromatic Euchromatic

Figure 1. LSM proteins silence heterochromatic reporters integrated as high-copy-number gene arrays, but not euchromatic reporters. (A) The integrated, high-copy-number heterochromatic reporter pkIs 1582 used in the genome-wide screen (Towbin et al. 2012). RNA interference (RNAi)-based derepression monitored in progeny of all stages by increased green fluorescent protein (GFP) fluorescence. $(B)$ An RNAi-based derepression screen was used to monitor derepression of pkIs 1582 (GFP) in embryos within young adult worms. The initial screen was reported in Towbin et al. (2012). (C) Fluorescence microscopy of pkIs1582 (GFP) derepression in L4 larvae treated with the indicated RNAi clones, versus control (mock/L4440). Bar, $100 \mu \mathrm{m}$. A + indicates relative intensity of GFP fluorescence. (D) Quantitation of GFP intensity of heterochromatic reporter pkIs 1582 in L1 larvae (GW306) following indicated RNAi by the worm sorter. L1 progeny were scored after RNAi of indicated genes, or control RNAi (mock: L4440). Shown are box plots of fluorescence intensity in arbitrary units (a.u.), with whiskers = 1st and 3rd quartiles; black lines, median; black circles, outliers. The notch around the median represents $95 \%$ confidence interval of the median. Quantification and statistical analysis were based on $n=375$ for each condition. (E) Quantitation of derepression of the heterochromatic (GW306) as well as the euchromatic (GW1108) reporters in L1 progeny under $l s m-6, l s m-7$, and control RNAi conditions, as in $D$. Numbers of L1 worms scored are $n=2000$ (GW306-mock), 1068 (GW306-lsm6), 613 (GW306-lsm7) and 875 (GW1108-mock), 1110 (GW1108-lsm6), 1026 (GW1108-lsm7), pooled from three independent experiments. Indicated $P$-values by two-tailed unpaired Student's $t$-test (n.s. =nonsignificant or $P>0.05$ ). Strains are described in Table 1. (Data in panels $C, D$ are found in different formats in Mattout et al. 2020. Reproduced with permission.) 
Table 1. Strains and reporters used in this study

\begin{tabular}{|c|c|c|c|}
\hline Strain & $\begin{array}{l}\text { Original } \\
\text { name/OC }\end{array}$ & Genotype & Reference \\
\hline GW1 & $\mathrm{N} 2^{\mathrm{a}}$ & Wild-type, Bristol isolate & \\
\hline GW76 & & gwIs4 [baf-1p::GFP-lacI::let-858 3'UTR; myo-3p::RFP] X & Meister et al. 2010 \\
\hline GW306 & NL2507 & pkIs 1582[let-858p::GFP:: let-858 3'UTR; rol-6(su1006)] V & Towbin et al. 2012 \\
\hline GW566 & & $\begin{array}{l}\text { gwIs39 [baf-1p::GFP-LacI::let-858 3'UTR; vit-5p::GFP] III; gwIs4 [baf-1p:: } \\
\text { GFP-lacI::let-858 3'UTR; myo-3p::RFP] X }\end{array}$ & Towbin et al. 2012 \\
\hline GW694 & & $\begin{array}{l}\text { gwIs85[his-72p::mcherry-set-25::his-72 3'UTR; unc-119(+)] unc-119(ed3) } \\
\text { ttTi5605 II }\end{array}$ & Towbin et al. 2012 \\
\hline GW299 & & gwIs 25 [tbb-1p::wmCherry-LacI::tbb-2 3'UTR unc-119(+)] unc-119(ed3) & Mattout et al. 2020 \\
\hline GW637 & & $\begin{array}{l}\text { met-2(n4256) set-25(n5021) III; gwIs4 [baf-1p::GFP-lacI::let-858 3'UTR; } \\
\text { myo-3p::RFP] X }\end{array}$ & Towbin et al. 2012 \\
\hline GW214 & & hpl-2(tm1489) III; gwIs4 [baf-1p::GFP-lacI::let-858 3'UTR; myo-3p::RFP] X & Towbin et al. 2012 \\
\hline GW468 & & $\begin{array}{l}\text { mes-2(bn11) unc-4(e120)/mnC1 dpy-10e1280 unc-52(e444)II; gwIs4[myo-3:: } \\
\text { RFP baf-1::GFP-lacI let-858] X. }\end{array}$ & Towbin et al. 2012 \\
\hline GW1004 & & lsm 4:GFP-FLAGx3 & Mattout et al. 2020 \\
\hline GW1109 & HW1390 ocx4 & lsm-8 (xe17 [myo2p::mcherry::unc54 3'UTR])IV & Mattout et al. 2020 \\
\hline GW1125 & ocx 6 & lsm-8 (xe17 [myo2p::mcherry::unc54 3'UTR])IV/nT1[qIs51](IV;V) & Mattout et al. 2020 \\
\hline GW1119 & ocx 6 & $\begin{array}{l}\text { lsm-8 (xe17 [myo2p::mcherry::unc54 3'UTR])IV/nT1[qIs51](IV;V); pkIs } 1582 \\
\quad \text { [let-858::GFP rol-6(su1006)]V }\end{array}$ & Mattout et al. 2020 \\
\hline GW1120 & ocx 6 & $\begin{array}{l}\text { lsm-8 (xe17 [myo2p::mcherry::unc54 3'UTR])IV; pkIs 1582[let-858::GFP rol-6 } \\
\quad(\text { su1006)] } \mathrm{V}\end{array}$ & Mattout et al. 2020 \\
\hline GW1148 & ocx6 & $\begin{array}{l}\text { met-2(n4256) set-25(n5021) III; lsm-8 (xe17 [myo2p::mcherry::unc54 3'UTR]) } \\
\quad I V / n T 1[q I s 51](I V ; V)\end{array}$ & Mattout et al. 2020 \\
\hline GW1080 & $\mathrm{VC} 2785^{*}$ ocx 5 & lsm-4\&ada-2(ok3151)/mln1[mls14dpy-10(e128)]II & Mattout et al. 2020 \\
\hline
\end{tabular}

hand, was detectable throughout worm development - that is, in larvae and in adult worms (L4 larvae shown in Fig. 1C; see also Mattout et al. 2020). This was also true for other RNAi hits from the initial screen-namely, sams-3 RNAi and mes-4 RNAi (Fig. 1C). Elevated GFP expression from the heterochromatic array after RNAi treatment was scored at all stages of worm development and in nearly every somatic cell type, except germline cells (see below). Given that Piwiinteracting RNAs (piRNAs) are known to contribute to silencing uniquely in germline cells, we hypothesize that the effect of $l \mathrm{sm}$ gene down-regulation may be masked by redundant silencing pathways, like piRNAs, that act specifically in the gonad. Alternatively, the LSM-mediated silencing pathway may not be functional in germ cells.

To obtain a quantitative value for reporter expression, we monitored the increase in GFP fluorescence intensity using the COPAS BioSort, which measures fluorescence on a worm-by-worm basis for hundreds to thousands of worms, using flow cytometry. Using the worm sorter, we compared the effects of $l s m-6, l s m-2 / g u t-2$, and $l s m-5$ RNAi with the knockdown of further shared subunits of the two LSM complexes: LSM-3, LSM-4, and LSM-7 (Fig. 1D). The RNAi clone we used against $l s m-3$ had several predicted targets; therefore, the results were inconclusive (data not shown). However, in case of both $l s m-4$ and lsm-7 RNAi, a significant $\left(P<10^{-13}\right)$ increase in average GFP fluorescence of twofold to fourfold was scored (see Mattout et al. 2020). The variable degrees of GFP derepression following RNAi against these LSM subunits likely stems from differences in RNAi efficiency.

Given that the LSM complex has been implicated in RNA processing (Kufel et al. 2004; Beggs 2005; Tharun
2009; Perea-Resa et al. 2012; Golisz et al. 2013; Cornes et al. 2015), it was possible that somatic cell derepression was specific to the reporter construct used in the screen. We ruled this out by monitoring the effects of $l s m-2, l s m$ 5 , or $l s m-6$ RNAi on the expression of four different heterochromatic reporters each integrated in the C. elegans genome as a large array (200-300 copies) at a single locus (Mattout et al. 2020). Each reporter carried a different promoter, gene, and 3' UTR, yet all showed reporter up-regulation following $l \mathrm{sm}-2, l \mathrm{sm}-5$, or lsm-6 RNAi (Mattout et al. 2020). In contrast, two euchromatic reporters, which were single-copy transgenes integrated into a nonheterochromatic region of the genome, showed no change in expression under the same conditions (data shown for GW1108, eft-3p::gfp cb-unc$119(+) \mathrm{chr}$ II in Fig. 1E). This was true for euchromatic reporters with both high and low levels of expression, indicating that LSM complex subunits are selectively involved in the repression of loci that have heterochromatic, but not euchromatic features. Derepression levels were comparable or greater than the loss of our positive control, MES-4, a histone H3K36 methyltransferase recovered in the original screen (Towbin et al. 2012), and persisted during somatic cell differentiation and in postmitotic cells.

We confirmed that the up-regulation of GFP in these worms indeed reflects changes on the mRNA level, rather than GFP protein synthesis or turnover. This was achieved by quantifying the reporter mRNA by quantitative polymerase chain reaction using reverse transcription (RTqPCR) following RNAi against various LSM subunits, notably $l s m-6$ or $l s m-7$ down-regulation (Mattout et al. 2020). 

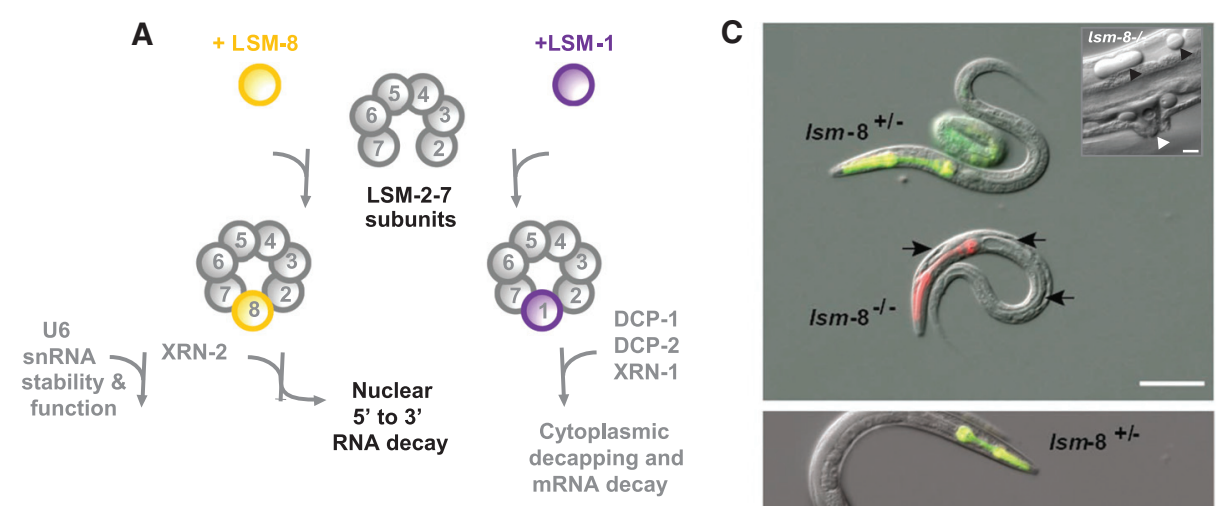

B
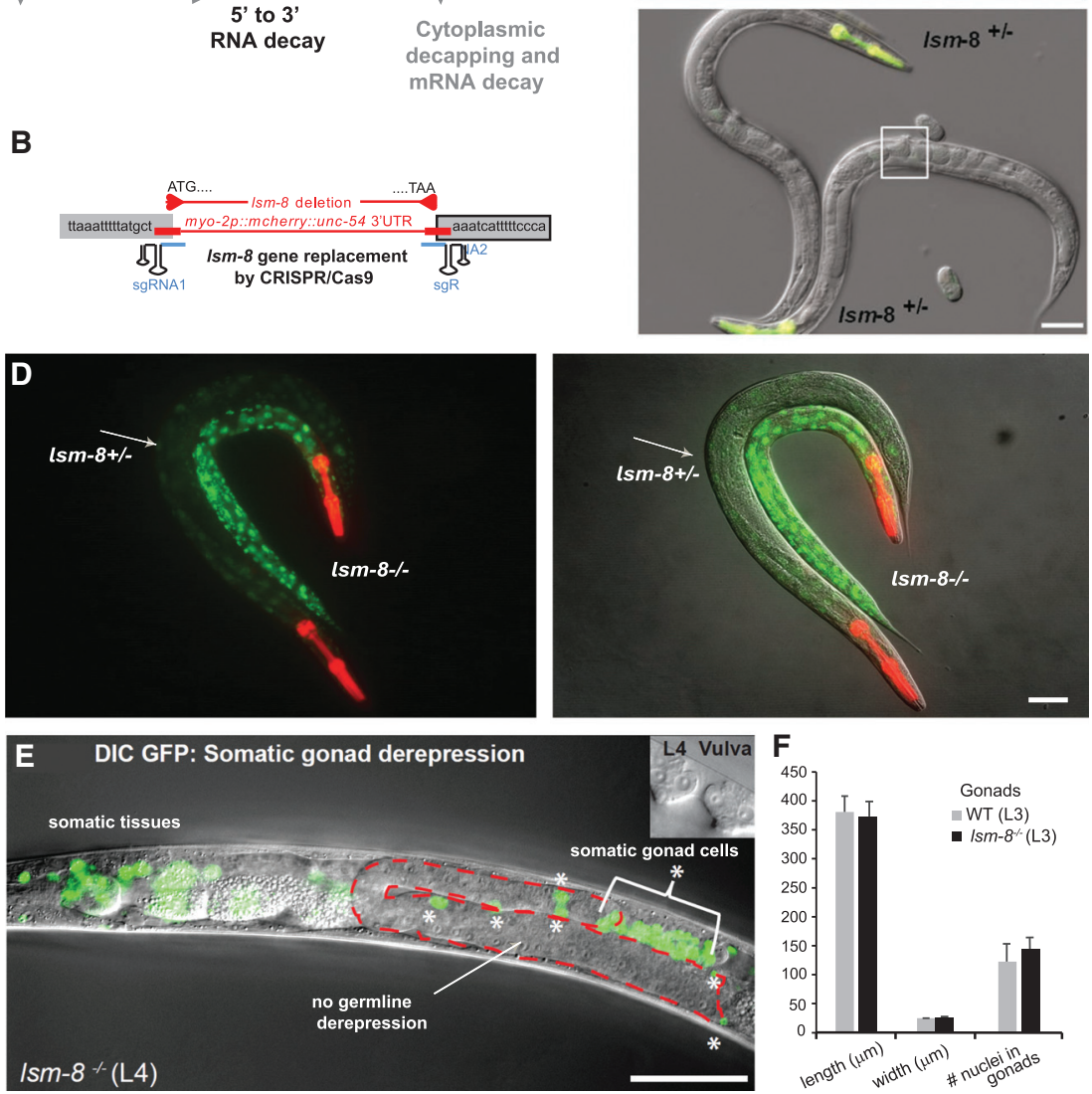

Figure 2. LSM-8 is required for heterochromatic reporter silencing, implicating the LSM2-8 complex. $(A)$ The two main LSM complexes and functions (Beggs 2005; Tharun 2009). (B) Schematic view of the lsm-8 deletion/gene replacement created by CRISPR-Cas9. (C) Differential interference contrast (DIC) images of L1/L2 larvae (upper panel) and young adults (lower panel) together with dual-channel fluorescence for $l s m-8^{+-}$(yellow pharynx in merge) and $l s m-8^{-/}$(red pharynx only) worms. Red and green channels are merged to distinguish heterozygous from homozygous worms. The inset in the upper panel shows the protruding vulva of an $l s m-8^{-/-}$adult (marked with white triangle) and the accumulating vesicles (black triangles). The normal vulva is boxed in the lower panel. Bars $=100 \mu \mathrm{m}$. $(D)$ Red and green fluorescence channels are merged and coupled with DIC images of one $l \mathrm{sm}-8^{-/-}$worm and one $l s m-8^{+/-}$heterozygote bearing the pkIs1582 reporter (GW1120). Note that this strain has no nT1 balancer with the green fluorescent pharynx marker. GFP fluorescence in nuclei throughout the $l s m-8^{-/}$worm is visible. Bar, $100 \mu \mathrm{m}$. (E) Merged DIC images with fluorescent images as in D. lsm- $8^{-/}$mutant L4 larvae bearing pkIs 1582 reporter is shown with fully developed gonad (encircled in red). The $l \mathrm{sm}-8^{-/-}$mutant is $100 \%$ sterile. Somatic nuclei and somatic cells of the gonad show GFP expression from the heterochromatic array. Bar, $50 \mu \mathrm{m}$. (This image also appeared in Mattout et al. 2020. Reproduced with permission.) (F) Quantification of the length, width, and gonad nuclei count from the DAPI staining of L3 WT and $l s m-8^{-/-}$larvae. $n=8$.

\section{LSM2-8 CONTRIBUTES TO HETEROCHROMATIC REPORTER SILENCING}

Studies in yeast and plants have established that there are two LSM complexes (LSM1-7 and LSM2-8) (Fig. 2A) that share six subunits, whereas the seventh subunit in each complex, LSM-1 or LSM-8, participates only in one (Beggs 2005; Tharun 2009). The two complexes also in- teract with distinct cofactors. RNAi against $l s m-1$, one of the nonshared subunits, did not derepress the heterochromatic reporter nor did the depletion of the $5^{\prime} \rightarrow 3^{\prime}$ exoribonucleases, XRN-1, which is reported to act together with LSM1-7 in the cytoplasm to mediate cytoplasmic RNA decay (Figs. 1D and 2A). On the other hand, RNAi against $x r n-2$, a nuclear RNase that is thought to degrade RNA together with LSM2-8 in budding yeast (Tharun 2009), 
did show significant heterochromatic reporter derepression (Fig. 1D). Null-allele worms lacking $l s m-1$ or the decapping factor $d c a p-2$ also failed to show array derepression (Mattout et al. 2020). This argued against a role for LSM1-7 in heterochromatic reporter silencing and suggested instead that LSM2-8 and an evolutionarily conserved nuclear $5^{\prime} \rightarrow 3^{\prime}$ exoribonuclease called XRN-2 (HsXRN2, SpDhp1, or ScRat1) are involved (Miki et al. 2016).

Because LSM-8, the only subunit unique for the nuclear LSM2-8 complex, was unresponsive to RNAi in our hands, it was essential to create a genomic deletion allele of the $l s m-8$ gene. A full $l s m-8$ deletion was generated by CRISPR-Cas9, and simultaneously we inserted a red fluorescent marker gene with pharynx-specific expression. We could track the null allele by fluorescence microscopy, but found that the homozygous $l s m-8^{-/-}$worms were $100 \%$ sterile. To propagate the $l s m-8$ deletion, the mutation was balanced with a green fluorescence-marked balancer $n T 1$ [qIs51], which is also expressed in the pharynx. With dual fluorescence, we could distinguish offspring that are heterozygous for $l s m-8^{-/+}$(i.e., that have red and green fluorescence in the pharynx) from those that are homozygous (lsm $8^{-1-}$; red pharynx only) (Fig. 2C). GFP fluorescence from the heterochromatic array was then monitored in red pharynx-marked homozygous, $l s m-8^{-/-}$ animals with or without (Fig. 1D) the $n T 1$ balancer. Indeed, deficiency for LSM-8 strongly derepressed the heterochromatic array, yielding GFP fluorescences at levels similar to those observed after $l s m-2, l \mathrm{sm}-5, l \mathrm{sm}-6$, or $l \mathrm{sm}$ 7 RNAi (Fig. 2D). Although mutant germline and gonad development up to L3 and L4 larval stages was similar to that in wild-type (N2) worms (Fig. 2E,F), there was no oocyte maturation in the $l s m-8^{-/-}$mutant. Nonetheless, we could monitor GFP derepression in all somatic tissues of larvae and adults, including the somatic cells of the gonad, but not in the germline itself (Fig. 2E).

Although the $l s m-8^{-/-}$animals can develop to adulthood, the adults have protruding vulva (see inset, Fig. $2 \mathrm{C}$, white arrowhead), show empty cavities or vacuoles in differentiated tissues (black triangle), and die prematurely (Mattout et al. 2020). Other $l s m$ mutants, such as $l s m-2$ and $l s m-5$, are phenotypically similar to $l s m-8$ mutants, with clear protruding vulva, the presence of vacuoles, and $100 \%$ sterility. Adult populations of the $l s m-8$ homozygous phenotype started dying after 6 days, whereas a similar loss of viability in wild-type worms would not occur before 10-12 days. Worms mutant for $l \mathrm{sm}-1 \mathrm{did}$ not present these phenotypes (Cornes et al. 2015), confirming the distinct roles played by the two LSM complexes.

\section{LSM2-8 IS REQUIRED TO MAINTAIN SILENT ENDOGENOUS HETEROCHROMATIN}

Although LSM2-8 was clearly implicated in heterochromatic array silencing, it was important to establish whether or not it also controls the expression of endogenous transcripts. The data sets for total RNA-seq of WT and homozygous $l s m 8^{-1-}$ sorted L3 and L1 larvae are presented in Mattout et al. (2020) and data are deposited with the NCBI Gene Expression Omnibus (GSE92851).
The $l s m-8$ data sets were compared with RNA-seq data from similarly sorted met-2 $2^{-1-}$ set- $25^{-1-}$ L3 larvae, which lack detectable $\mathrm{H} 3 \mathrm{~K} 9$ methylation (Towbin et al. 2012), and from L3 worms of the triple mutant, met- $2^{-/-}$ set- $25^{-/-} ; l s m 8^{-/-}$, to determine if the LSM2-8 silencing pathways would be epistatic with the classic H3K9 methylation-mediated repression. In each case, the mutant and wild-type worms were sorted by fluorescence (nongreen pharynx indicating homozygosity) and by size, to generate a uniform population of L3 stage larvae. Mutant and wildtype $\mathrm{L} 1$ populations were also examined following sorting for L1 size larvae (Mattout et al. 2020). For the L3 populations, shifts in developmental timing arising during the sorting process were compensated by comparing matched replicates by making use of a characteristic, temporal fluctuation in gene expression characterized by the Großhans laboratory (Hendriks et al. 2014). With developmentally matched mutants and control larvae, we analyzed the RNA-seq data using edgeR, to determine the genes that changed significantly in each of three genetic backgrounds and WT strains (Mattout et al. 2020). Two or three analytical criteria were used, with the most stringent being the combined criteria of a false discovery rate (FDR) $<$ 0.05 and a fold change $(\mathrm{Fc})$ in average RNA level $>4$ (Mattout et al. 2020). In Figure 3A, we highlight genes with average altered expression rates of $\mathrm{Fc}>4$ only.

We found that the deletion of $l s m-8$ led to the strong and reproducible up-regulation of 122 genes (FDR $<0.05$ and $\mathrm{Fc}>4$ ), with very few genes being down-regulated (Mattout et al. 2020). With less stringent cutoff values, even higher numbers of loci were selectively derepressed in $l s m-8^{-/-}$(Fig. 3A): Only FDR $<0.05$ identified 147 genes up-regulated; only Fc $>4$ identified 204 genes; and only $\mathrm{Fc}>2$ identified 1332 genes. Interestingly, a significant number of transcripts sensitive to $l s m-8^{-/-}$were involved in the innate immune response and the regulation of cell shape, but they did not reflect a single developmental stage. Genes derepressed in the mutant in L1 larvae overlapped only partially $(5 \%-10 \%$ based on the thresholds used) with those derepressed in the L3 stage, and among those overlapping, many are involved in the innate immune response (Mattout et al. 2020). We confirmed the lsm $8^{-1-}$ larvae results by performing RNA-seq experiments on the $l s m-4$ mutant (Fig. 4A). The patterns of genes derepressed were overlapping but not identical, in $l s m-4$ and $l s m-8$ mutants (Fig. 4B). This is to be expected, given that $l_{s m}-4$ also eliminates the activity of the LSM1-7 complex as well as the LSM2-8. Indeed, the distribution of LSM-4-GFP is diffuse in both cytoplasm and nucleus, and in the nucleus it appears to be excluded from foci that bind the H3K9me3 histone methyltransferase (HMT), SET-25 (labeled with mCherry, Fig. 4C).

In yeast and plants, RNA-binding complexes feed back to establish $\mathrm{H} 3 \mathrm{~K} 9$ methylation on centromeric and other heterochromatin (Buhler et al. 2007; Vasiljeva et al. 2008; Keller et al. 2012; Zofall et al. 2012; Yamanaka et al. 2013; Egan et al. 2014; Chalamcharla et al. 2015; Tucker et al. 2016). It was therefore of interest to compare RNAseq changes provoked by the loss of LSM2-8 with loss of canonical heterochromatin marks, $\mathrm{H} 3 \mathrm{~K} 9 \mathrm{me} 2$ or me3. We 
A RNA-seq: Gene expression changes

* up-regulated $(\mathrm{Fc}>4)$
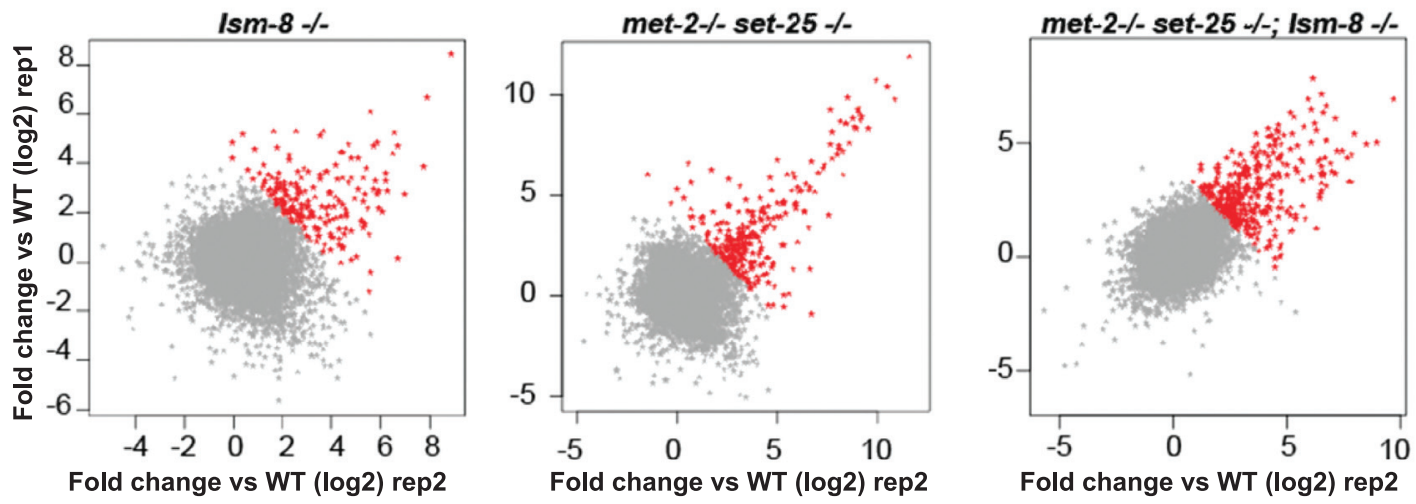

B

RNA-seq: $m e t-2^{-/}$set-25/- vs Ism-8 ${ }^{-/-}$

C
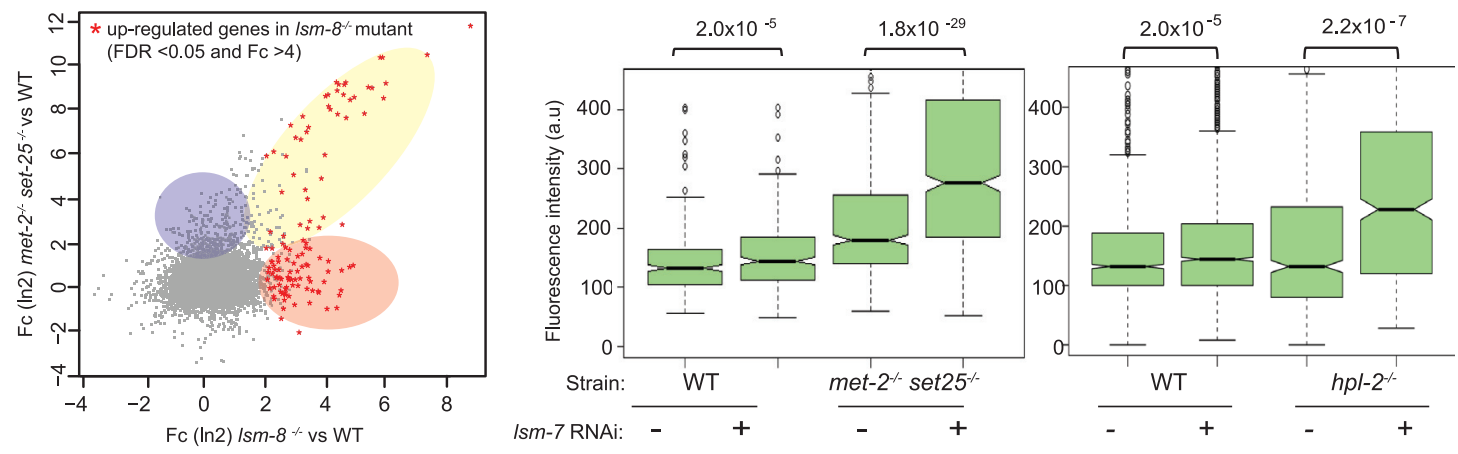

D

E

Associated histone marks:

F
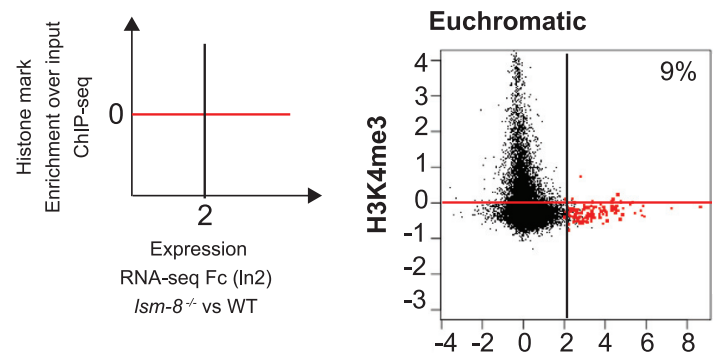

Heterochromatic

Abs. expression (In2)
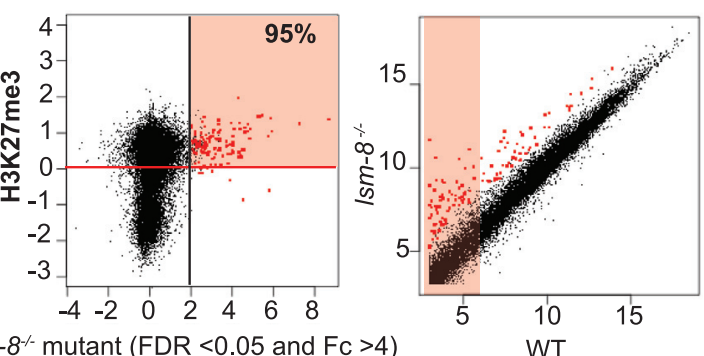

Figure 3. LSM2-8 silences hundreds of genes and $95 \%$ of these carry the Polycomb mark H3K27me3. (A) Deletion of $l \mathrm{sm}-8\left(\mathrm{lsm}-8^{-/-}\right)$ derepresses significantly more than 200 genes $(\mathrm{Fc}>4)$. Relative gene expression profiles are shown as scatter plots, with fold change ( $\mathrm{Fc}$ $>4$ ) for two RNA-seq replicas of L3 sorted worms of the indicated genotype versus WT. Each dot corresponds to a gene. The original RNA-seq data are available at NCBI Gene Expression Omnibus number GSE92851. The same data set was analyzed in Mattout et al. (2020), but with (FDR $<0.05$ and $\mathrm{Fc}>4)$. (B) Scatter plot comparing the relative gene expression between the $l s m-8$ ( $x$-axis) and the $m e t-$ 2 set-25 double mutant ( $y$-axis). Common up-regulated genes are shaded yellow; $36 \%$ of genes up-regulated in the $l s m-8$ mutant (FDR $<$ 0.05 and $\mathrm{Fc}>4)$ are also up-regulated ( $\mathrm{FDR}<0.05$ and $\mathrm{Fc}>4$ ) in the met-2 set-25 mutant. $l$ sm- $8^{-/-}$-specific up-regulated genes are shaded in pink; met- $2^{-/-}$set- $25^{-/-}$-specific are in blue. (This panel is reproduced from Figure $3 \mathrm{C}$ in Mattout et al. 2020 with permission.) (C) LSM2-8-mediated silencing of the heterochromatic reporter is independent of H3K9 methylation. Quantitation of derepression of GFP expressed from the gwIs 4 heterochromatic reporter in L1 progeny in WT and met-2 set-25 mutant genotypes, after control or $l s m-7$ RNAi. Quantitation of GFP derepression expressed from the gwIs4 heterochromatic reporter in L1 progeny in WT and met-2 set-25 mutant genotypes, respectively, from strains GW76 and GW637, after control or $l \mathrm{sm}$-7 RNAi. $P$-values indicated; two-tailed unpaired $t$ test. Quantification and statistical analyses were based on $n=1460,2399,1593$, and 1189 worms for conditions indicated from left to right, pooled from three independent experiments. $N=339,1004,426,673$ same after $l s m-7$ RNAi in the $h p l-2^{-/-}$mutant background. $(D, E)$ Scatter plots compare the average gene expression changes in $l s m-8^{-1-}$ worms $\left(x\right.$-axis in $\log _{2}$, RNA-seq L3 stage) versus enrichment for the indicated euchromatic or heterochromatic histone modification ( $y$-axis in $\log _{2}$, ModEncode data of WT L3 stage). Up-regulated genes (FDR $>0.05$ and $\mathrm{Fc}>4$ ) in the $l s m-8^{-/-}$mutant are in red to the right of the black line, and genes enriched for the histone mark are above the red line (enriched over input). \% indicates genes in upper right zone. (The panels in $E$ are reproduced from Fig. 4B of Mattout et al. 2020, with permission.) (F) Scatter plot of absolute gene expression $\left(\log _{2}\right)$ of $l s m-8^{-/-}$versus WT. Red dots as in $E$ and each dot corresponds to a gene. Values $<6\left(\log _{2}\right)$ are considered as very low expression: $6\left(\log _{2}\right)$ corresponds to about 60 RNAseq reads per gene, whereas $10\left(\log _{2}\right)$ corresponds to about 1000 reads per gene. Most $l s m-8^{-/-}$derepressed genes have low steady-state level expression in WT worms but moderate expression levels in the mutant (pink shading). (This panel is reproduced from Fig. $4 \mathrm{C}$ of Mattout et al. 2020, with permission.) 


\section{A RNA-seq: Ism4-I- vs WT (L1)}

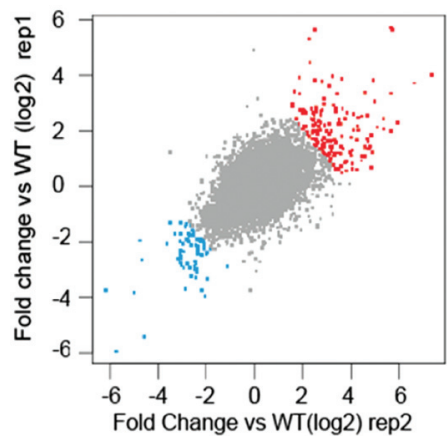

- up-regulated genes in $/ s m-4-/$ - mutant $(\mathrm{FDR}<0.05$ and $\mathrm{Fc}>4)$

In down-regulated genes in $/ \mathrm{sm}-4 \%$ mutant $(\mathrm{FDR}<0.05$ and $\mathrm{Fc}<4)$
B RNA-seq Ism4-/- vs /sm-8-/-

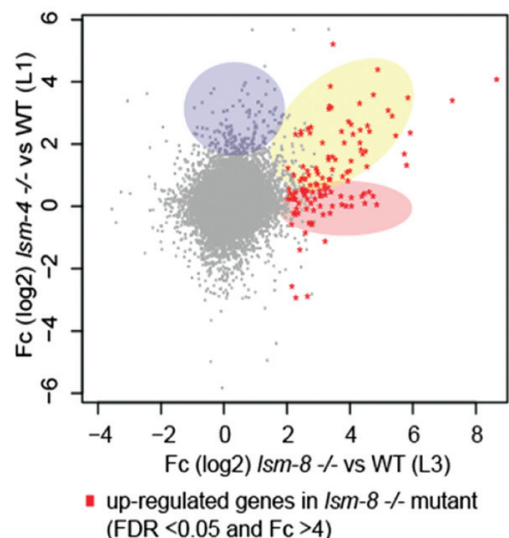

C LSM-4-GFP and H3K9me3 HMT SET-25-mCherry imaging
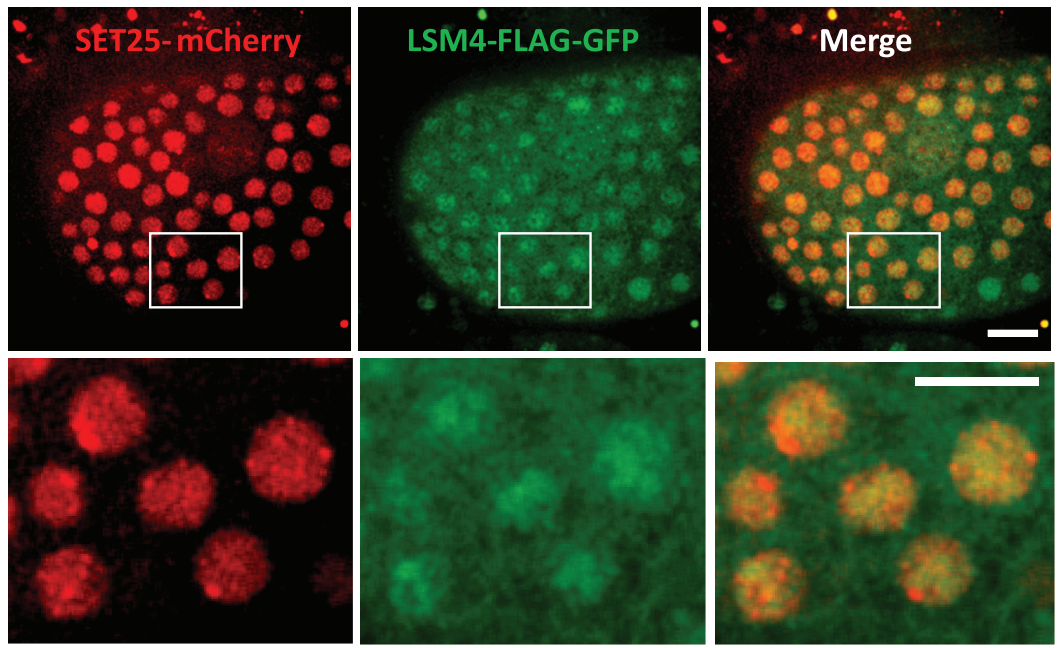

Figure 4. $l s m-8$ and $l s m-4$ deletions derepress overlapping gene sets in L1 and L3 larvae. (A) Deletion of $l s m-4\left(l s m-4^{-/-}\right)$leads to L1 larval arrest and derepresses more than 100 genes significantly. Relative gene expression profiles are shown as scatter plots of fold change $(\mathrm{Fc})$ in $\log _{2}$ of two RNA-seq replicas of L1 stage worms, $l \mathrm{sm}-4^{-1-}$ versus WT. Each dot corresponds to a gene. The class of genes that were most significantly affected control body morphogenesis $(P=0.0072)$. $(B)$ Scatter plot comparing the relative gene expression between the $l s m-8$ ( $x$-axis) and the $l s m-4$ mutant ( $y$-axis). Common up-regulated genes are shaded yellow; $53 \%$ of genes up-regulated in $l s m-8(\mathrm{FDR}<0.05$ and $\mathrm{Fc}>4)$ are also up-regulated to some extent $\left(50 \%\right.$ increase) in $l \mathrm{sm}-4$. Genes up-regulated in $l s m-8^{-l-}$ only are shaded pink, and those in $l s m-4^{-1-}$ only in blue. $(C) Z$-projection of live confocal images of embryonic progeny issued from the cross between the strain (GW1004) expressing LSM-4-Flag-GFP (GFP, green channel) and the strain (GW694) SET-25, the H3K9me3 HMT, fused to mCherry. The merged image shows no significant overlap on the brightest foci of SET-25. The boxed area is enlarged to show nuclear foci in bottom panels. Bars, $10 \mu \mathrm{m}$.

found that $36 \%$ of the genes derepressed because of the loss of H3K9 methylation (met-2 $2^{-/}$set- $25^{-/}$) overlapped with those depressed in the $l \mathrm{sm}-8^{-1-}$ mutant at the L3 stage (Fig. 3B; Mattout et al. 2020). To test the additivity of the LSM2-8 and H3K9me-mediated repression pathways, we compared the RNA-seq data of the single mutants with the triple mutant $\left(l \mathrm{sm} 8^{-/}\right.$; met- $2^{-1}$ - set- $25^{-/}$) (Fig. 3A) and found that there were clear subsets of up-regulated genes that were unique to either the $l s m-8$ or the met- 2 set-25 mutant, and that genes showing mild up-regulation upon loss of either $l s m-8$ or met- 2 set-25 ( $\mathrm{Fc} \sim 2)$ were more strongly expressed when combined (FDR $<0.05$ and $\mathrm{Fc}>4$ ) (Mattout et al. 2020). Thus, the LSM2-8 pathway of silencing for endogenous loci is distinct from that mediated by $\mathrm{H} 3 \mathrm{~K} 9 \mathrm{me} 2 / 3$, even though some genes are targeted by both pathways. Consistently using the heterochromatic reporter pkIS1582, we could show that $l s m-7$ RNAi derepresses in an additive fashion with the loss of either H3K9me3 (met-2 set-25) or the loss of the H3K9me3 reader, HPL-2 (Fig. 3C).

\section{LSM2-8 TARGETS POLYCOMB-MARKED GENES}

To ask what the $l s m-8$-sensitive genes have in common, we compared our RNA-seq data to the normalized ChIP- 
seq data of common histone modifications generated by ModEncode (Fig. 3D-E). In worms, as in most organisms, H3K4me2, H3K4me3, and H3K27ac are associated with active genes (Liu et al. 2011; Wenzel et al. 2011; Ho et al. 2014), whereas H3K9me2/3 and H3K 27 me 3 are repressive histone marks that colocalize with heterochromatin (Wenzel et al. 2011). The genes that were up-regulated in the $l s m-8^{-1-}$ mutant were depleted for active marks (H3K4me3) (Fig. 4E) in WT L3 larvae, and showed no particular enrichment for $\mathrm{H} 3 \mathrm{~K} 9 \mathrm{me} 2$ nor for $\mathrm{H} 3 \mathrm{~K} 9 \mathrm{me} 3$ (Mattout et al. 2020). Strikingly, however, 95\% of genes that were derepressed by loss of LSM- 8 were enriched for the repressive Polycomb mark, H3K27me3 (Fig. 3E). This was true not only for strongly up-regulated genes, but also for genes that were mildly up-regulated upon loss of LSM8 (less than fourfold) and for windows of $500 \mathrm{bp}$ across the genome. Thus, the overriding feature of genes or domains silenced by LSM2-8 was their enrichment for H3K27me3.

Consistent with the fact that H3K27me3 is the hallmark of Polycomb-repressed genes (Liu et al. 2011; Margueron and Reinberg 2011; Grossniklaus and Paro 2014; Conway et al. 2015), the absolute steady state level of the $l s m-8$ sensitive mRNAs was very low or almost undetectable in WT larvae (Fig. 3F). Although H3K27me3 is deposited in C. elegans by a PRC2-like complex comprising MES-2/ $\mathrm{E}(\mathrm{z}) / \mathrm{Ezh} 2, \mathrm{MES}-3$, and MES-6/Esc (Ketel et al. 2005; Yuzyuk et al. 2009; Gaydos et al. 2014) and mediates cell type-specific repression of developmentally regulated genes, in worms H3K27me3 and H3K9me3 ChIP-seq signals colocalize at many sites across the $C$. elegans genome (Ho et al. 2014). Interestingly, based on the ModEncode data sets from WT L3 larvae, $83 \%$ of the genes that are reproducibly enriched for $\mathrm{H} 3 \mathrm{~K} 9 \mathrm{me} 3$ are also enriched for $\mathrm{H} 3 \mathrm{~K} 27 \mathrm{me} 3$, and $41 \%$ of the genes enriched for $\mathrm{H} 3 \mathrm{~K} 27 \mathrm{me} 3$ also carry H3K9me3. Among the genes upregulated by $l s m-8$ mutation, we find that $40 \%-43 \%$ also carry H3K9me3 (Mattout et al. 2020). Because this rate is the same as the genome-wide coincidence of paired K9/ K27 methylation, it appears that nucleosomes or domains bearing both repressive marks are not significantly enriched among lsm-8 targets. Most likely, H3K9me2 or me3 marks are irrelevant, for LSM2-8 recruitment or silencing.

\section{LSM2-8 SILENCES THROUGH RNA DEGRADATION}

Given that the LSM2-8 complex is known to stabilize and bind U6 snRNA in yeast and plants (Beggs 2005; Perea-Resa et al. 2012; Zhou et al. 2014), and that the LSM2-8 complex coprecipitates both with a factor involved in U6 snRNA stability (Ruegger et al. 2015) and with U6 snRNA itself in C. elegans (Mattout et al. 2020), we analyzed the RNA-seq data for splicing defects. We compared the exon-exon junction reads in the $l s m-8$ RNA-seq data with those from WT worms, to see if LSM2-8-mediated silencing coincides with defects in splicing, but we found irregular splicing events at only 18 exon-exon junctions out of 134,836 splice junctions
$(<0.02 \%)$ (Mattout et al. 2020). Thus, impaired splicing is most likely not the cause of $l s m-8^{-/}$-triggered derepression.

If $l \mathrm{sm} 8^{-1-}$ worms reduced the expression of PRC2 or PRC1 homologs, then it would follow that histone H3K27me3 levels would be reduced, and Polycomb-target genes would be derepressed. By checking the relevant mRNA levels, we could rule this out as a mechanism (Mattout et al. 2020). Nonetheless, to explore the relationship of LSM2-8-mediated silencing to H3K27me3, we treated the heterochromatic reporter-bearing $l \mathrm{sm}-8^{-/-}$ worms with RNAi against either mes-2 (EZH2) or $x r n$ 2 . As controls, we combined the $l s m-8$ mutant with RNAi against $l s m-7$ for epistasis and with mes-4 (H3K36me HMT) or set-25 (H3K9me HMT), which both show additivity with $l s m-8$ (Fig. 5A). Although $x r n-2$ RNAi is epistatic with the $l s m-8$ background, the depletion of mes-2 increased reporter expression, albeit less than that observed for set-25 or mes-4 RNAi (Fig. 5A). This argues that Polycomb-mediated silencing is not exclusively dependent on LSM-8. Again, this can be explained by the fact that $\mathrm{H} 3 \mathrm{~K} 27 \mathrm{me} 3$ is known to repress on the level of transcription. The strong epistasis observed with XRN-2, on the other hand, argued that LSM2-8 may silence through RNA turnover or degradation (Fig 5A). Comparison of RNA-seq data from worms treated with $x r n-2$ RNAi at L4 stage (Miki et al. 2016) and $l s m-8$ mutant worms at L3 confirms significant overlap of up-regulated endogenous genes (Fig. 5B). Indeed, 71\% of the genes upregulated by $l s m-8^{-1-}$ were also up-regulated by $x r n-2$ RNAi (Fig. 5B, yellow) and 95\% of those genes are enriched for $\mathrm{H} 3 \mathrm{~K} 27 \mathrm{me} 3$. Again, this argues that LSM-8 and XRN-2 function on the same pathway. Nonetheless, a subset $(<1 / 3)$ of LSM2-8 target genes (pink) were not affected by the down-regulation of XRN-2. It is unclear if those reflect experimental differences or a subset of genes silenced by LSM2-8 through another mechanism.

\section{LSM2-8 ACTS TOGETHER WITH RNA DEGRADATION ENZYMES}

To find other genes implicated in this potential nuclear RNA decay function, we tested candidate cofactors for heterochromatic reporter derepression and examined their genetic relationship with $l s m-8^{-/-}$. Heterochromatic array derepression at the L1 stage was monitored by GFP expression, following a targeted RNAi screen against genes that are co-regulated with LSM2-8 subunits and/or functionally implicated in RNA processing-namely, RNA Pol II subunits (rpb-12, rpb-7) and the type II poly(A) binding protein ( $p a b p-2)$. Like $x r n-2$ RNAi, RNAi against $r p b-12, r p b-7$, or $p a b p-2$ on their own derepress the heterochromatic reporter (Fig. 5A), whereas the depletion of six additional genes that are implicated in aspects of RNA metabolism does not (F49C12.11, T13F2.2, cgh-1the human ortholog of DDX6, B0495.8, C50D2.8, and pab-2 - the human ortholog of PABPC1) (Mattout et al. 2020). Important, nonetheless, is the epistasis between derepression by $x r n-2$ RNAi, $r p b-12, r p b-7$, and $p a b p-2$ 
A

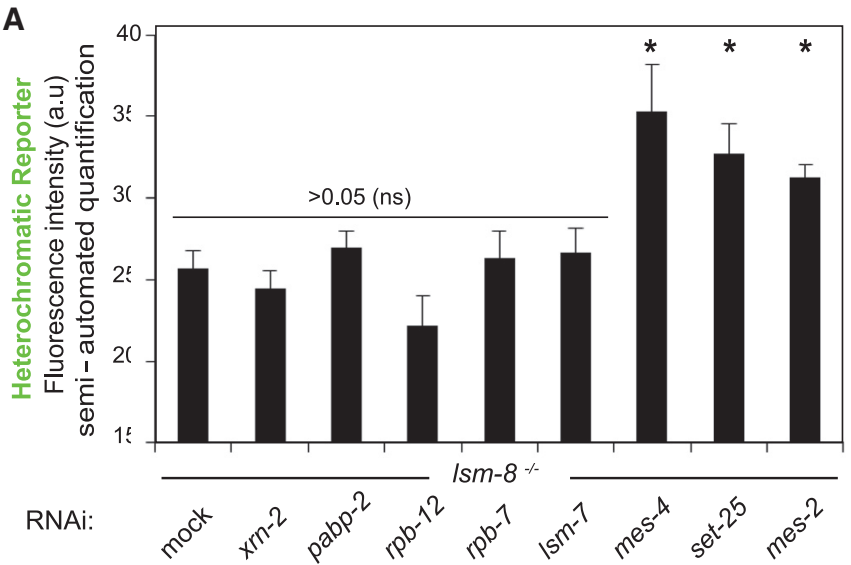

B

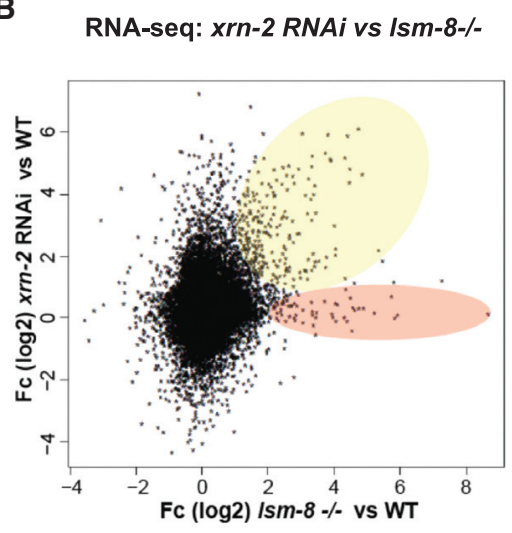

C

RNA decay assay
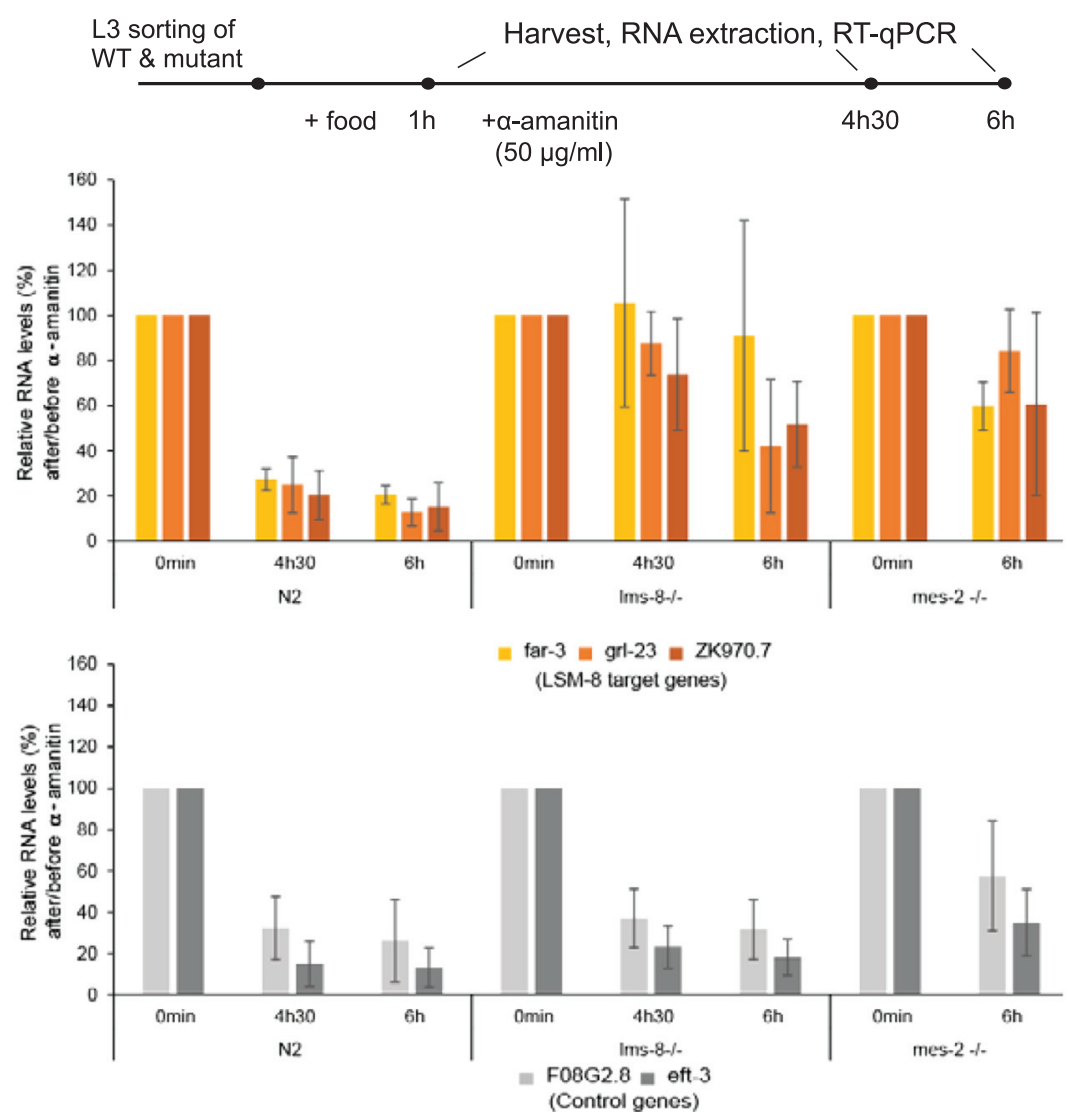

Figure 5. XRN-2 and other RNA factors show epistasis with $l s m-8$ deficiency for heterochromatic array derepression. (A) RNAi experiments were performed in the $l s m-8^{-/-}$(GW1119) strain. The GFP derepression of the heterochromatic reporter pkIs1582 was quantified by semiautomated fluorescence microscopy on homozygous $l s m-8^{-1-}$ progeny under indicated RNAi conditions. $P$-values (Students $t$-test) are indicated: $(*) P<0.005$. This indicates additivity between the $l s m-8$ derepression and derepression by the RNAi used. Bars $=$ S.E.M., $N=2, n=55,45,22,11,10,23,25,25,85$. (These data are reproduced from Fig. $6 \mathrm{~F}$ of Mattout et al. 2020, with permission.) (B) Scatter plot comparing relative gene expression changes of $l s m-8^{-1-}$ L3 larvae and $x r n-2$ RNAi treated L4 (Miki et al. 2016). Common up-regulated genes are shaded yellow; $71 \%$ of genes up-regulated in the $l s m-8$ mutant $(\mathrm{Fc}>4$ ) are also up-regulated to some extent (50\% increase) in $x r n-2$ depleted worms. $l \mathrm{sm}-8^{-/-}$-specific up-regulated genes are shaded pink. (This panel is reproduced from Fig. 6A of Mattout et al. 2020, with permission.) (C) WT and $l \mathrm{sm}-8^{-\prime-}$ worms were sorted, refed with OP50 in liquid culture for $1 \mathrm{~h}$ at room temperature, and treated with $50 \mu \mathrm{g} / \mathrm{mL}$ final concentration of $\alpha$-amanitin, which inhibits Pol II and Pol III transcription. RNA was isolated at time $0,4.5$, and $6 \mathrm{~h}$, as indicated for each independent experiment. RNA levels of three transcripts affected by LSM-8 and two control transcripts (expression not affected by LSM-8) were determined by RT-qPCR and normalized to 18S rRNA levels, which are insensitive to $\alpha$-amanitin. The value at $0 \mathrm{~h}$ is defined as $100 \%$. Error bars represent standard deviation of the mean from at least three independent experiments. (Data are reproduced from Extended Data Fig. 8A in Mattout et al. 2020, with permission.) 
and the $l s m-8$ mutant. In conclusion, PABP-2, RPB-12, and RPB-7 may act with XRN-2 on the LSM2-8-mediated silencing pathway.

\section{LSM2-8 CONTRIBUTES TO THE DEGRADATION OF mRNAs FROM POLYCOMB-MARKED GENES}

The cooperation between the RNA-binding LSM2-8 complex and XRN-2 suggests that LSM2-8 may silence genes by triggering mRNA degradation. It would follow that $l s m-8$ deletion might increase the RNA stability of specific LSM2-8-sensitive transcripts in vivo because of a failure to degrade these RNAs by XRN-2. This was confirmed for the mRNAs of three endogenous $l s m-8$ sensitive genes-far-3, grl-23, and ZK970.2-by monitoring mRNA stability after the addition of $\alpha$-amanitin, an inhibitor of RNA Pol II and Pol III elongation. The rate of mRNA decay was monitored at the L3 stage of sorted WT and $l s m-8^{-1-}$ worms over $6 \mathrm{~h}$ using RTqPCR. RNA levels were normalized to that of the $18 \mathrm{~S}$ rRNA. Deletion of $l s m-8$ delayed the turnover of far-3, grl-23, and ZK970.2 mRNAs, whereas the mRNA and pre-mRNA of two control genes (eft-3 and F08G2.8) showed no change in turnover rates (Fig. 5C; Mattout et al. 2020). Consistently, transcript levels from these lsm-8-sensitive genes, far-3 and ZK970.2, were also strongly up-regulated in $x r n-2$ RNAi treated worms as well, with a fold change in $\log _{2}$ of 4.9 and 4.0, respectively (Mattout et al. 2020). The decay of the three $l s m-8$-sensitive mRNAs was also attenuated in the Polycomb-deficient strain (mes-2) (Mattout et al. 2020). Thus, our data suggest that MES-2-mediated repression of LSM-8 regulated genes acts at least in part through mRNA degradation (Mattout et al. 2020).

\section{LSM2-8 ACTION FEEDS BACK TO MAINTAIN H3K27me3 LEVELS}

Although mes- 2 and $l s m-8$ depletion appeared to be additive with respect to derepression, one cannot conclude that the LSM2-8 complex acts independently of MES-2 and $\mathrm{H} 3 \mathrm{~K} 27 \mathrm{me} 3$, especially considering the striking correlation of LSM-8-sensitivity with H3K27me3 (Fig. 3E). We initially tested for global changes in repressive epigenetic marks, H3K9me3 and H3K27me3, by immunostaining of embryos following $l s m-7$ RNAi. However, we found no global drop in staining (Fig. 6A). Nonetheless, when we performed quantitative chromatin immunoprecipitation (ChIP) for H3K27me3 on genes that are sensitive to lsm8 ablation (i.e., genes silenced by the LSM2- 8 complex in WT worms), we observed a significant decrease (>50\%) in H3K27me3 levels (Fig. 7B). This was not detected for $l s m-$ 8 -insensitive H3K27me3-marked genes. This suggests that the LSM2-8 complex selectively feeds back to maintain H3K27me3 levels, either directly or indirectly, at a subset of H3K27me3-silenced loci. The fact that it is not detectable by immunostaining argues that H3K27me3 lev-

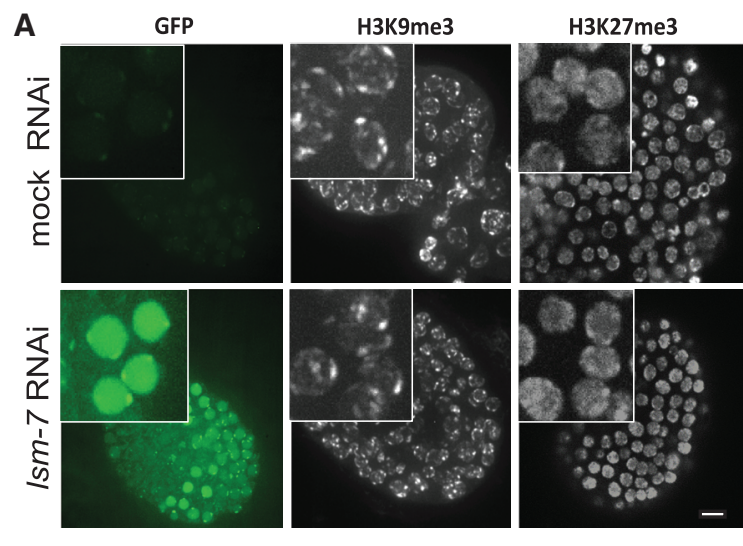

\section{B H3K27me3 ChIP}

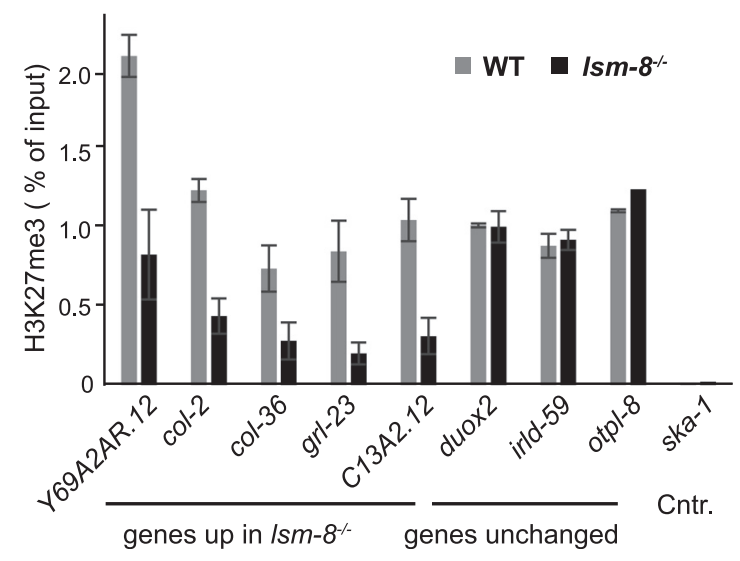

Figure 6. LSM2-8 helps maintain full $\mathrm{H} 3 \mathrm{~K} 27 \mathrm{me} 3$ levels on silenced loci. (A) Z-projection of confocal images of wild-type

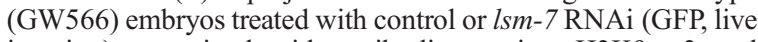
imaging) or stained with antibodies against $\mathrm{H} 3 \mathrm{~K} 9 \mathrm{me} 3$ and $\mathrm{H} 3 \mathrm{~K} 27 \mathrm{me} 3$, as indicated. Nuclei are enlarged in the insets. Bar, $5 \mu \mathrm{m}$. (B) H3K27me3 ChIP-qPCR on target genes in WT and $l s m-8^{-1-}$ worms. Three categories of genes were assessed. Five genes were monitored that are up-regulated in $l s m-8^{-1-}$ worms and enriched for H3K27me3 mark in WT (lsm-8 target genes), three genes were monitored that are enriched for the H3K27me3 mark in WT but do not change in expression in the lsm-8 mutant (nontarget genes), and the control category is represented by ska-1, a gene not enriched for H3K27me3 in WT and with no expression change (control). $N=3, n=3$, bars (mean $\pm \mathrm{S}$. E.M.). (Data appear in Fig. 7A of Mattout et al. 2020 and are reproduced with permission.)

els may not be affected globally, although many Polycomb targets do show significant derepression.

\section{CONCLUSION}

We have shown that in nematodes a conserved nuclear RNA-binding complex, LSM2-8, contributes to full repression of heterochromatic reporters and genomic regions that carry the H3K27me3 epigenetic mark. A related, cytoplasmic complex, LSM1-7, appears to have no specific role in heterochromatic silencing. Unlike heterochromatin-linked RNA degradation pathways in plants and fission yeast, LSM-2-8-mediated repression is inde- 


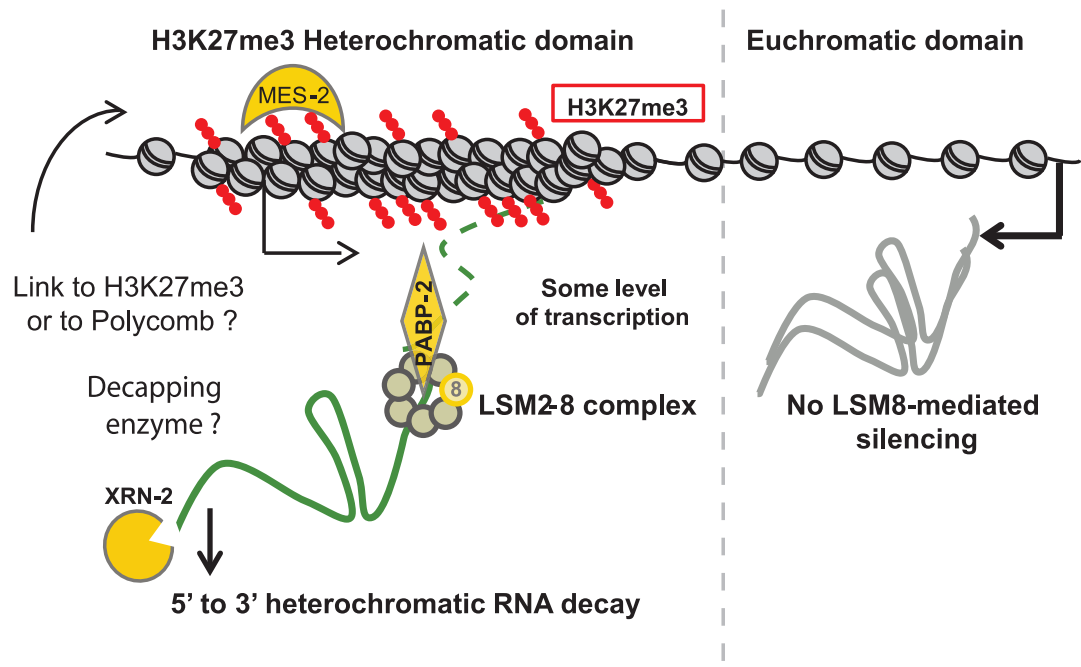

Figure 7. Model for the LSM2-8 pathway of silencing. We showed that the LSM-8-mediated silencing pathway makes use of XRN-2 ribonuclease and involves other factors, such as PABP-2 (H.s: PABPN1). We hypothesize that RNA arising from H3K27me3 genomic regions that are controlled by the LSM2-8 complex acquire a specific feature during transcription (e.g., a specific structure, RNA modification, 3' UTR, poly(A/U) tail, or specific RNA-binding protein(s)), which allows recognition and processing by LSM2-8. LSM2-8-mediated silencing also feeds back to regulate H3K27me3 levels on LSM-8-regulated genes, although it is unclear if the interaction with PRC2 complex is direct (dotted arrow). LSM-2-8-mediated silencing of H3K27me3-bound regions defines a new mechanism for selective post-/cotranscriptional silencing for facultative heterochromatin through RNA decay.

pendent of and additive with $\mathrm{H} 3 \mathrm{~K} 9 \mathrm{me} 3$ and $\mathrm{H} 3 \mathrm{~K} 9 \mathrm{me}$ HMTs. Our data suggest that the LSM2-8 complex acts primarily on RNA stability, selectively mediating the decay of transcripts from H3K27me3-tagged genes through the 5'-3' exoribonuclease XRN-2. The pathway does not, however, involve XRN-1 nor the decapping enzyme DCAP-2. The LSM2-8-mediated silencing occurs at Polycomb H3K27me3-marked genes, with only 40\% of the LSM2-8 targets also carrying H3K9me3. This represents the stochastic frequency of $\mathrm{H} 3 \mathrm{~K} 27 \mathrm{me} 3$ and $\mathrm{H} 3 \mathrm{~K} 9 \mathrm{me} 3$ coincidence on genes in L3 stage larvae, suggesting that $\mathrm{H} 3 \mathrm{~K} 27 \mathrm{me} 3$ and not $\mathrm{H} 3 \mathrm{~K} 9 \mathrm{me} 3$ is the recognition signal for mRNA degradation. As sketched in our model in Figure 7, we propose that the LSM2-8 complex mediates gene silencing primarily through the exonuclease XRN-2, which would degrade the transcripts from a subset of H3K27me3-marked genes either co- or posttranscriptionally. This represents a novel pathway for Polycomb-mediated gene silencing in animals, which appears to act in addition to transcriptional repression.

The fact that only a subset of transcripts arising from H3K27me3-marked genes is found to be significantly derepressed in $l s m-8$ mutant worms suggests that there may be additional criteria that provide specificity for LSM2-8 control. Using a less stringent cutoff for derepression in the $l s m-8$ RNA-seq data set (Mattout et al. 2020), we find many more genes up-regulated, and $>93 \%$ still bear H3K27me3. It is important to note that the derepression of tissue-specific transcripts (i.e., those that are expressed in only a few cells of larvae or adult worms) will not be detectable by our RNA-seq methods that monitor RNA in whole worm extracts. This was confirmed to be the case for one HOX gene called egl-5, which is known to be regulated by MES-2 (EZH2) (Mattout et al. 2020). By analyzing the cell type-specific derepression of egl-5 using fluorescence microscopy, we were able to show similar levels of derepression of this gene in male tail cells, in both $l s m-8$ and mes-2 mutants (Mattout et al. 2020), although its derepression level by RNA-seq was only $\sim 1.5$-fold.

The molecular basis of LSM2-8 specificity for H3K27me3 provides a framework for future studies exploring how the epigenetic information can be transferred from the chromatin to an RNA-binding complex. We propose that the RNAs arising from $\mathrm{H} 3 \mathrm{~K} 27$ me3-marked genomic regions are recognized and bound selectively by the LSM2-8 complex, and that this likely requires a feature that is acquired during transcription from that locus-for example, a specific structure, RNA modification, cap, a poly-A/U tail, or specific RNA-binding protein(s) recognized by LSM2-8. It is important to note that we scored a drop in $\mathrm{H} 3 \mathrm{~K} 27 \mathrm{me} 3$ specifically on LSM2-8-sensitive genes in $l s m-8^{-/-}$animals, even though the overall staining level of H3K27me3 appeared unchanged (Fig. 6). This suggests that RNA degradation helps maintain H3K27me3 levels. In other words, transcriptional repression by this epigenetic mark may be transferred from the RNA-binding complex LSM2-8 to chromatin.

An RNA decay-mediated pathway involving the cofactors PABP-2, RPB-12, and RBP-7 appears to function independently of DACP-2 and LSM-1 and additively with HPL-2 and the H3K9me3 HMT, SET-25. The epistasis with type II poly(A) binding protein (PABP-2) (Hurschler et al. 2011) (HsPABPN1 and SpPab2) is particularly interesting, because it appears to regulate $3^{\prime}$ UTR and poly(A) tail length in the nucleus (Kuhn et al. 2009). Moreover, it binds nascent RNAs early during the elongation step 
(Lemieux and Bachand 2009; Beaulieu et al. 2012). Given that the LSM2-8 complex is known to bind to the 3 ' oligo(U) tail of the U6 snRNA (Zhou et al. 2014), as well as the $3^{\prime}$ poly $(\mathrm{A}+$ ) tail of nuclear RNAs (Kufel et al. 2004), PABP-2 may regulate its specificity by modulating the 3' end of mRNAs at H3K27me3-marked domains. The participation of RNA Pol II subunits RPB-7 and -12 in LSM2-8-mediated gene silencing is also suggestive of an involvement of RNA Pol II in silencing, just as the Schizosaccharomyces pombe RBP-7 homolog has been implicated in centromeric repeat RNAi-directed silencing (Djupedal et al. 2005). Interestingly, fission yeast lacks H3K27me3; thus, the link to chromatin status may have evolved at a later stage. In $S$. cerevisiae, the same subunit plays a different, but direct role in Pat1/Lsm1-7-mediated mRNA decay in the cytoplasm (Lotan et al. 2007; Haimovich et al. 2013). One speculative scenario, based on functions described in other organisms, is that $r p b-7$ and $r p b-12$ RNA Pol II subunits might be loaded on LSM2-8-regulated transcripts to signal to that these should be degraded by XRN-2. An unknown decapping enzyme(s) may also be involved, which would allow RNA degradation by XRN-2 in the nucleus.

We conclude that facultative heterochromatin in metazoans can be silenced through a mechanism of selective transcript degradation and not only by transcriptional repression. LSM2-8 may mediate gene silencing by linking a specific epigenetic state to transcript degradation, adding an additional layer of control over differentiation and development.

\section{ACKNOWLEDGMENTS}

The accession number for the RNA-seq data is NCBI Gene Expression Omnibus GSE92851. Some strains were provided by the Caenorhabditis Genetics Center (CGC), which is funded by the National Institutes of Health Office of Research Infrastructure Programs (P40 OD010440). We thank I. Katiç, the Friedrich Miescher Institute Genomics and Microscopy facilities, and M. Bühler, H. Großhans, and W. Filipowicz for discussions. The authors acknowledge the support of Marie Curie Intra-European grant (no. PIEF-GA-2010-276589) and the Swiss National Science Foundation (SNF) Marie-Heim Vögtlin grant (nos. PMPDP3_151381 and PMPDP3_168717) to A.M.; SNF grant (no. 310030B_156936) to S.M.G.; and support of the Novartis Research Foundation. This project received funding from the European Research Council under the European Union's Horizon 2020 Research and Innovation programme (Epiherigans_-grant agreement no. 743312 to S.M.G.).

\section{REFERENCES}

Beaulieu YB, Kleinman CL, Landry-Voyer A-M, Majewski J, Bachand F. 2012. Polyadenylation-dependent control of long noncoding RNA expression by the poly (A)-binding protein nuclear 1. PLoS Genet 8: e1003078. doi:10.1371/journal.pgen .1003078

Beggs JD. 2005. Lsm proteins and RNA processing. Biochem Soc Trans 33: 433-438. doi:10.1042/BST0330433
Bühler M. 2009. RNA turnover and chromatin-dependent gene silencing. Chromosoma 118: 141-151. doi:10.1007/s00412008-0195-Z

Bühler M, Haas W, Gygi SP, Moazed D. 2007. RNAi-dependent and -independent RNA turnover mechanisms contribute to heterochromatic gene silencing. Cell 129: 707-721. doi:10 $.1016 /$ j.cell.2007.03.038

Chalamcharla VR, Folco HD, Dhakshnamoorthy J, Grewal SI. 2015. Conserved factor Dhp1/Rat1/Xrn2 triggers premature transcription termination and nucleates heterochromatin to promote gene silencing. Proc Natl Acad Sci 112: 1554815555. doi:10.1073/pnas.1522127112

Conway E, Healy E, Bracken AP. 2015. PRC2 mediated H3K27 methylations in cellular identity and cancer. Curr Opin Cell Biol 37: 42-48. doi:10.1016/j.ceb.2015.10.003

Cornes E, Porta-De-La-Riva M, Aristizábal-Corrales D, BrokateLlanos AM, García-Rodríguez FJ, Ertl I, Díaz M, Fontrodona L, Reis K, Johnsen R, et al. 2015. Cytoplasmic LSM-1 protein regulates stress responses through the insulin/IGF-1 signaling pathway in Caenorhabditis elegans. RNA 21: 1544-1553. doi:10.1261/rna.052324.115

Djupedal I, Portoso M, Spåhr H, Bonilla C, Gustafsson CM, Allshire RC, Ekwall K. 2005. RNA Pol II subunit Rpb7 promotes centromeric transcription and RNAi-directed chromatin silencing. Genes Dev 19: 2301-2306. doi:10.1101/gad .344205

Egan ED, Braun CR, Gygi SP, Moazed D. 2014. Post-transcriptional regulation of meiotic genes by a nuclear RNA silencing complex. RNA 20: 867-881. doi:10.1261/rna.044479.114

Gaydos LJ, Wang W, Strome S. 2014. Gene repression. H3K27me and PRC2 transmit a memory of repression across generations and during development. Science 345: 15151518. doi:10.1126/science. 1255023

Golisz A, Sikorski PJ, Kruszka K, Kufel J. 2013. Arabidopsis thaliana LSM proteins function in mRNA splicing and degradation. Nucleic Acids Res 41: 6232-6249. doi:10.1093/nar/ gkt296

Gonzalez-Sandoval A, Towbin Benjamin D, Kalck V, Cabianca DS, Gaidatzis D, Hauer MH, Geng L, Wang L, Yang T, Wang $\mathrm{X}$, et al. 2015. Perinuclear anchoring of H3K9-methylated chromatin stabilizes induced cell fate in C. elegans embryos. Cell 163: 1333-1347. doi:10.1016/j.cell.2015.10.066

Grossniklaus U, Paro R. 2014. Transcriptional silencing by Polycomb-group proteins. Cold Spring Harb Perspect Biol 6: a019331. doi:10.1101/cshperspect.a019331

Haimovich G, Choder M, Singer RH, Trcek T. 2013. The fate of the messenger is pre-determined: a new model for regulation of gene expression. Biochim Biophys Acta 1829: 643-653. doi:10.1016/j.bbagrm.2013.01.004

Hendriks G-J, Gaidatzis D, Aeschimann F, Großhans H. 2014. Extensive oscillatory gene expression during C. elegans larval development. Mol Cell 53: 380-392. doi:10.1016/j.molcel .2013.12.013

Ho JW, Jung YL, Liu T, Alver BH, Lee S, Ikegami K, Sohn KA, Minoda A, Tolstorukov MY, Appert A, et al. 2014. Comparative analysis of metazoan chromatin organization. Nature 512: 449-452. doi:10.1038/nature13415

Hurschler BA, Harris DT, Grosshans H. 2011. The type II poly(A)-binding protein PABP-2 genetically interacts with the let-7 miRNA and elicits heterochronic phenotypes in Caenorhabditis elegans. Nucleic Acids Res 39: 5647-5657. doi:10 $.1093 / \mathrm{nar} / \mathrm{gkr} 145$

Keller C, Adaixo R, Stunnenberg R, Woolcock KJ, Hiller S, Bühler M. 2012. HP1 ${ }^{\text {Swi6 }}$ mediates the recognition and destruction of heterochromatic RNA transcripts. Mol Cell 47: 215-227. doi:10.1016/j.molcel.2012.05.009

Ketel CS, Andersen EF, Vargas ML, Suh J, Strome S, Simon JA. 2005. Subunit contributions to histone methyltransferase activities of fly and worm Polycomb group complexes. Mol Cell Biol 25: 6857-6868. doi:10.1128/MCB.25.16.6857-6868 .2005

Kufel J, Bousquet-Antonelli C, Beggs JD, Tollervey D. 2004. Nuclear pre-mRNA decapping and 5 ' degradation in yeast 
require the Lsm2-8p complex. Mol Cell Biol 24: 9646-9657. doi:10.1128/MCB.24.21.9646-9657.2004

Kühn U, Gündel M, Knoth A, Kerwitz Y, Rüdel S, Wahle E. 2009. Poly (A) tail length is controlled by the nuclear poly(A)-binding protein regulating the interaction between poly(A) polymerase and the cleavage and polyadenylation specificity factor. $J$ Biol Chem 284: 22803-22814. doi:10 $.1074 /$ jbc.M109.018226

Lemieux C, Bachand F. 2009. Cotranscriptional recruitment of the nuclear poly (A)-binding protein Pab2 to nascent transcripts and association with translating mRNPs. Nucleic Acids Res 37: 3418-3430. doi:10.1093/nar/gkp207

Liu T, Rechtsteiner A, Egelhofer TA, Vielle A, Latorre I, Cheung M-S, Ercan S, Ikegami K, Jensen M, Kolasinska-Zwierz P, et al. 2011. Broad chromosomal domains of histone modification patterns in C. elegans. Genome Res 21: 227-236. doi:10 $.1101 /$ gr. 115519.110

Lotan R, Goler-Baron V, Duek L, Haimovich G, Choder M. 2007. The Rpb7p subunit of yeast RNA polymerase II plays roles in the two major cytoplasmic mRNA decay mechanisms. J Cell Biol 178: 1133-1143. doi:10.1083/jcb.200701165

Margueron R, Reinberg D. 2011. The Polycomb complex PRC2 and its mark in life. Nature 469: 343-349. doi:10.1038/ nature 09784

Mattout A, Gaidatzis D, Padeken J, Schmid C, Aeschlimann F, Kalck V, Gasser SM. 2020. LSM2-8 and XRN-2 contribute to the silencing of $\mathrm{H} 3 \mathrm{~K} 27 \mathrm{me} 3$-marked genes through targeted RNA decay. Nature Cell Biol doi:10.1038/s41556020-0504-1

Meister P, Towbin BD, Pike BL, Ponti A, Gasser SM. 2010. The spatial dynamics of tissue-specific promoters during $C$. elegans development. Genes Dev 24: 766-782. doi:10.1101/ gad.559610

Miki TS, Carl SH, Stadler MB, Großhans H. 2016. XRN2 autoregulation and control of polycistronic gene expression in $\mathrm{Cae}$ norhabditis elegans. PLoS Gen 12: e1006313. doi:10.1371/ journal.pgen.1006313

Perea-Resa C, Hernández-Verdeja T, López-Cobollo R, del Mar Castellano M, Salinas J. 2012. LSM proteins provide accurate splicing and decay of selected transcripts to ensure normal Arabidopsis development. Plant Cell 24: 4930-4947. doi:10 $.1105 /$ tpc. 112.103697

Rüegger S, Miki TS, Hess D, Großhans H. 2015. The ribonucleotidyl transferase USIP-1 acts with SART3 to promote U6 snRNA recycling. Nucleic Acids Res 43: 3344-3357. doi:10 $.1093 / \mathrm{nar} / \mathrm{gkv} 196$

Saksouk N, Simboeck E, Déjardin J. 2015. Constitutive heterochromatin formation and transcription in mammals. Epigenetics Chromatin 8: 3. doi:10.1186/1756-8935-8-3
Tharun S. 2009. Roles of eukaryotic Lsm proteins in the regulation of mRNA function. Int Rev Cell Mol Biol 272: 149-189. doi:10.1016/S1937-6448(08)01604-3

Towbin BD, Meister P, Pike BL, Gasser SM. 2010. Repetitive transgenes in C. elegans accumulate heterochromatic marks and are sequestered at the nuclear envelope in a copy-numberand lamin-dependent manner. Cold Spring Harb Symp Quant Biol 75: 555-565. doi:10.1101/sqb.2010.75.041

Towbin BD, González-Aguilera C, Sack R, Gaidatzis D, Kalck V, Meister P, Askjaer P, Gasser SM. 2012. Step-wise methylation of histone $\mathrm{H} 3 \mathrm{~K} 9$ positions heterochromatin at the nuclear periphery. Cell 150: 934-947. doi:10.1016/j.cell.2012.06.051

Trojer P, Reinberg D. 2007. Facultative heterochromatin: is there a distinctive molecular signature? Mol Cell 28: 1-13. doi:10 $.1016 /$ j.molcel.2007.09.011

Tucker JF, Ohle C, Schermann G, Bendrin K, Zhang W, Fischer T, Zhang K. 2016. A novel epigenetic silencing pathway involving the highly conserved $5^{\prime}-3^{\prime}$ exoribonuclease Dhp1/ Rat1/Xrn2 in Schizosaccharomyces pombe. PLoS Gen 12: e1005873. doi:10.1371/journal.pgen.1005873

Vasiljeva L, Kim M, Terzi N, Soares LM, Buratowski S. 2008. Transcription termination and RNA degradation contribute to silencing of RNA polymerase II transcription within heterochromatin. Mol Cell 29: 313-323. doi:10.1016/j.molcel.2008 .01 .011

Wang J, Jia ST, Jia S. 2016. New insights into the regulation of heterochromatin. Trends Genetics 32: 284-294. doi:10.1016/j .tig.2016.02.005

Wenzel D, Palladino F, Jedrusik-Bode M. 2011. Epigenetics in C. elegans: facts and challenges. Genesis 49: 647-661. doi:10 1002/dvg.20762

Yamanaka S, Mehta S, Reyes-Turcu FE, Zhuang F, Fuchs RT, Rong Y, Robb GB, Grewal SI. 2013. RNAi triggered by specialized machinery silences developmental genes and retrotransposons. Nature 493: 557-560. doi:10.1038/nature11716

Yuzyuk T, Fakhouri TH, Kiefer J, Mango SE. 2009. The polycomb complex protein mes-2/E $(z)$ promotes the transition from developmental plasticity to differentiation in C. elegans embryos. Dev Cell 16: 699-710. doi:10.1016/j.devcel.2009 .03 .008

Zhou L, Hang J, Zhou Y, Wan R, Lu G, Yin P, Yan C, Shi Y. 2014. Crystal structures of the Lsm complex bound to the 3' end sequence of U6 small nuclear RNA. Nature 506: 116-120. doi:10.1038/nature12803

Zofall M, Yamanaka S, Reyes-Turcu FE, Zhang K, Rubin C, Grewal SI. 2012. RNA elimination machinery targeting meiotic mRNAs promotes facultative heterochromatin formation. Science 335: 96-100. doi:10.1126/science. 1211651 


\section{$\$_{\text {CSH }}^{\infty}$ Cold Spring Harbor Symposia SYMPOSIA on Quantitative Biology}

\section{A Nuclear RNA Degradation Pathway Helps Silence Polycomb/H3K27me3-Marked Loci in Caenorhabditis elegans}

Anna Mattout, Dimos Gaidatzis, Véronique Kalck, et al.

Cold Spring Harb Symp Quant Biol published online April 29, 2020

Access the most recent version at doi:10.1101/sqb.2019.84.040238

$\mathbf{P}<\mathbf{P} \quad$ Published online April 29, 2020 in advance of the print journal.

Creative This article is distributed under the terms of the

Commons http://creativecommons.org/licenses/by-nc/4.0/, which permits reuse and

License redistribution, except for commercial purposes, provided that the original author and source are credited.

Email Alerting Receive free email alerts when new articles cite this article - sign up in Service the box at the top right corner of the article or click here.

Advance online articles have been peer reviewed and accepted for publication but have not yet appeared in the paper journal (edited, typeset versions may be posted when available prior to final publication). Advance online articles are citable and establish publication priority; they are indexed by PubMed from initial publication. Citations to Advance online articles must include the digital object identifier (DOIs) and date of initial publication.

To subscribe to Cold Spring Harbor Symposia on Quantitative Biology go to: http://symposium.cshlp.org/subscriptions 